\title{
Transformation of Symmetrization Order to Nuclear-Spin Magnetization by Chemical Reaction and Nuclear Magnetic Resonance
}

\author{
C. Russell Bowers and Daniel P. Weitekamp \\ Arthur Amos Noyes Laboratory of Chemical Physics, California Institute of Technology, Pasadena, California 91125
}

(Received 2 July 1986)

\begin{abstract}
A method of obtaining very large nuclear-spin polarizations is proposed and illustrated by densityoperator calculations. The prediction is that chemical reaction and rf irradiation can convert the scalar parahydrogen state into polarization of order unity on the nuclear spins of the products of molecularhydrogen addition reactions. A means of extending the resultant sensitivity enhancement to other spins is proposed in which the transfer of order occurs through population differences not associated with magnetization.
\end{abstract}

PACS numbers: $33.25 .-\mathrm{j}, 35.20 . \mathrm{Bm}$

At ambient temperatures the equilibrium magnetization for an ensemble of nuclear spins in the applied magnetic field of a magnetic-resonance spectrometer is less than $10^{-4}$ of the value that would be obtained were all the spins parallel. Thus, enhancements of signal amplitude by several orders of magnitude are available if large nonequilibrium magnetizations can be obtained. Under certain conditions this is possible by a coupling of the nuclear-spin ordering to electron-spin degrees of freedom. In this Letter we propose a fundamentally different means of obtaining very large nonequilibrium nuclear-spin magnetizations. It may be viewed as a coupling, through a chemical reaction, of molecular rotation to nuclear-spin magnetization and relies on the strict correlation between rotational and nuclear-spin states which arises in molecules with identical nuclei from the symmetrization postulate of quantum mechanics. ${ }^{1}$ The concept is illustrated here algebraically and numerically for the simplest and most practical case, involving molecular addition reactions of parahydrogen followed by $\mathrm{rf}$ irradiation and detection near the proton Larmor frequency.

The quantum states of molecular hydrogen, $\mathrm{H}_{2}$, in both gaseous and condensed phases are divisible into two manifolds, orthohydrogen $\left(o-\mathrm{H}_{2}\right)$ and parahydrogen $(p-$ $\mathrm{H}_{2}$ ), with interconversion between them occurring at a rate usually far slower than the rate of equilibration within each manifold. The requirement that the overall molecular wave function be antisymmetric with respect to exchange of the two nuclei restricts the rovibrational states associated with each manifold in a well-known way. $^{2}$ In particular the lowest-energy state is in the para manifold and is separated from the lowest ortho states by a rotational energy quantum equivalent to $190 \mathrm{~K}$, allowing preparation of essentially pure $p-\mathrm{H}_{2}$, which can then be warmed up without a change of spin state.

Our concern here is with the nuclear-spin degrees of freedom only. The nuclear-spin density operator for $p$ $H_{2}$ is $\gamma(0)=\frac{1}{4}-I_{1} \cdot I_{2}$. The operators $I_{1}$ and $I_{2}$ are the spin angular momenta for the two hydrogen protons.
Within this spin space, the system is in a pure state and thus its spin entropy is zero. Since the ideal of maximum spin magnetization also corresponds to a zeroentropy state, the condensation to $p-\mathrm{H}_{2}$ is, in a thermodynamic sense, a step in the right direction. However, $p-\mathrm{H}_{2}$ has no spin angular momentum and thus no NMR signal. Evidently, the symmetry of the $\mathrm{H}_{2}$ molecule must be broken to result in a molecule which can be seen by NMR. We show here that addition of $p-\mathrm{H}_{2}$ to another molecule [Fig. 1(a)], followed by interaction of the spins both between one another and with a timedependent magnetic field, will provide the necessary transformation, converting much of the initial spin order into a very large nonequilibrium spin magnetization.

In most cases of interest, the other reactant will also

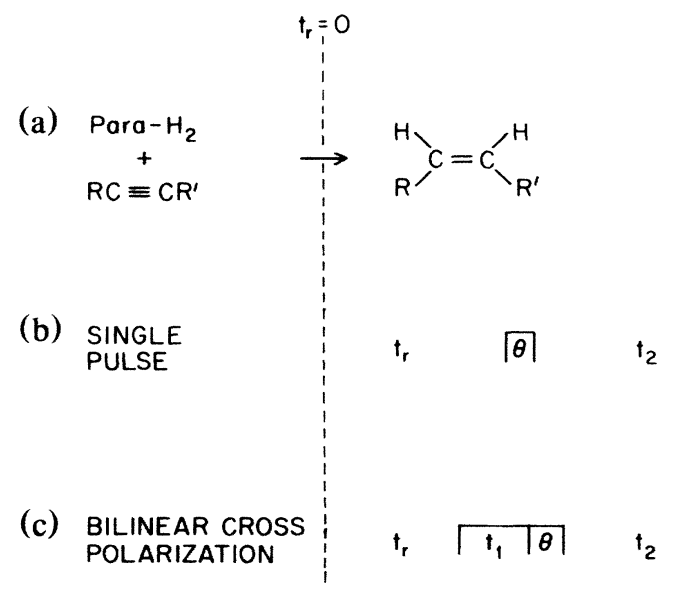

FIG. 1. Sequence of events for conversion of the spin order of parahydrogen to magnetization. The origin of time is the addition of molecular hydrogen to a suitable acceptor, as shown, for example, in (a). In (b) a brief mixing pulse of nutation angle $\theta$ at the proton Larmor frequency is delivered at a time $t_{r}$ after the reaction to elicit development of transverse magnetization during $t_{2}$. In (c) the mixing pulse is preceded by a cross-polarization sequence of length $t_{1}$ to share the spin order with weakly coupled resonances. 
contain nuclear spins. We assume for clarity that these other spins are initially completely disordered, their density operator being $\lambda(0)=1 / d_{\lambda}$, where $d_{\lambda}$ is the dimension (number of kets) needed to describe them. Both $\gamma(0)$ and $\lambda(0)$ are quasistationary states; they commute with the time-independent, motionally averaged, spin Hamiltonian of the separated reactants and change only on the time scale of the relevant spin-lattice processes. Suppose that the reactants are suddenly combined forming a new system with rotating-frame spin Hamiltonian $H$ and consider the subsequent unitary evolution. The sudden approximation gives the initial condition of the combined system as the product of the reactant density operators $^{3}: \quad \sigma(0)=\gamma(0) \lambda(0)$. The formation of the product will normally create chemical shifts and spinspin couplings such that $H$ does not commute with $\sigma(0)$.

Before exploring these dynamics case by case, it is valuable to extract some global features. First, it is clear that only tensor-operator components with zero projection (populations and zero-quantum coherence ${ }^{4}$ ) appear in the density operator following the reaction [period $t_{r}$ of Fig. 1(b)], since this quantum number is conserved in the assumed high field of the NMR spectrometer. Thus no transverse magnetization can spontaneously develop. Similarly, longitudinal magnetization is conserved during this time and must remain zero. Some perturbation is therefore necessary to evoke a net magnetization. A conceptually and experimentally simple possibility is the application of a coherent rf pulse at the Larmor frequency of one or more of the spins [Fig. 1(b)]. There will still be no spin magnetization immediately after a pulse brief enough to be described as a simple rotation operator. In summary, any observable response will depend in an oscillatory way on the time $t_{r}$ between formation of the product and the pulse and will develop from zero during the detection period $t_{2}$ after the pulse.

In order to focus attention separately on the first of these two dynamic problems, it is useful to calculate an average over the detection period. A useful measure is the signal energy per repetition,

$$
E\left(t_{r}\right)=C \int_{0}^{\infty}\left|S\left(t_{r}, t_{2}\right)\right|^{2} d t_{2},
$$

where the complex signal amplitude proportional to transverse magnetization is understood to be $S\left(t_{r}, t_{2}\right)$ $=\operatorname{Tr}\left[\sigma\left(t_{r}, t_{2}\right) I_{+}\right]$and all the invariant instrumental and system factors are collected in the constant $C$. A convenient point of reference is the signal energy $E_{0}$ that would be observed after a $\pi / 2$ pulse on a hypothetical product molecule having all $N$ protons fully polarized. The results for the various calculations involving $p-\mathrm{H}_{2}$ addition reactions will be expressed as the dimensionless ratio

$$
R\left(t_{r}\right)=E\left(t_{r}\right) / E_{0}
$$

Figure 2 shows numerical calculations of this quantity for the case of Fig. 1(b) where a single of pulse at the
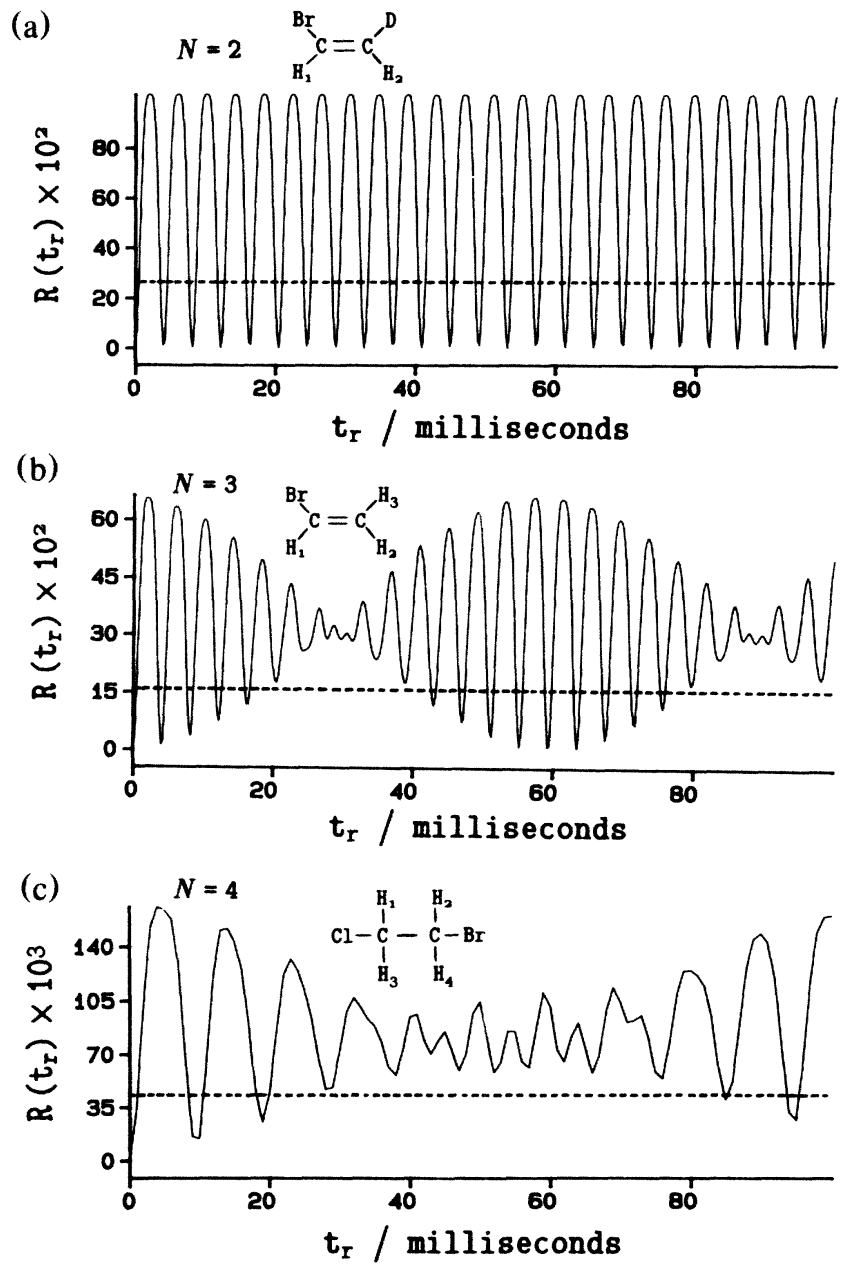

FIG. 2. Dependence of the signal energy on the delay between the reaction and a $\theta=n / 4$ rf pulse. The molecules (parameters in hertz) are (a) cis-1-deuterio-2-bromoethylene $\left(J_{12}=7.2, \quad v_{z 1}=-132, \quad v_{z 2}=112\right)$; (b) bromoethylene $\left(J_{12}\right.$ $=7.2, \quad J_{13}=15.0, \quad J_{23}=-1.7, \quad v_{z 1}=112, \quad v_{z 2}=-132, \quad v_{z 3}$ $=-200)$; (c) 1-bromo-2-chloroethane $\left(J_{1,2}=5.97, J_{14}=9.03\right.$, $\left.J_{13}-J_{24}=0.95, v_{z 1}=-33.33, v_{z 2}=71.7\right)$. The chemical shifts correspond to a proton Larmor frequency of $500 \mathrm{MHz}$. The vertical scales are relative to the signal energies that would be seen for perfect paramagnetic ordering subjected to a $\pi / 2$ pulse. The dashed lines indicate the level expected when the ensemble of product molecules is formed incoherently.

Larmor frequency separates the time since reaction $t_{r}$ from the detection period $t_{2}$. If we set the nutation angle $\theta=\pi / 4$, three simple molecules are considered that might be formed by molecular addition of hydrogen. The only relevant spins are those of protons, the other nuclei being taken as spinless, rapidly relaxing, or otherwise decoupled. The dynamics in Fig. 2 is illuminated by an analytical derivation for the case $N=2[\lambda(0)=1]$. With the weak-coupling Hamiltonian (in radians/second)

$$
H=-\omega_{z 1} I_{z 1}-\omega_{z 2} I_{z 2}+\bar{J}_{12} I_{z 1} I_{z 2}
$$


we obtain

$$
\sigma\left(t_{r}\right)=\frac{1}{4}-I_{z 1} I_{z 2}-\left(I_{x 1} I_{x 2}+I_{y 1} I_{y 2}\right) \cos \left(\omega_{z 1}-\omega_{z 2}\right) t_{r}+\left(I_{x 1} I_{y 2}-I_{y 1} I_{x 2}\right) \sin \left(\omega_{z 1}-\omega_{z 2}\right) t_{r} .
$$

Application of the $\theta$ pulse followed by further free evolution with detection gives the signal during $t_{2}$ as

$$
\begin{aligned}
S\left(t_{r}, \theta, t_{2}\right)=-\frac{1}{8}\left\{( a - i b ) \left[\exp \left(i\left(\omega_{z 1}+\frac{1}{2} \bar{J}_{12}\right) t_{2}\right)\right.\right. & \left.-\exp \left(i\left(\omega_{z 1}-\frac{1}{2} \bar{J}_{12}\right) t_{2}\right)\right] \\
& \left.-(a+i b)\left[\exp \left(i\left(\omega_{z 2}+\frac{1}{2} \bar{J}_{12}\right) t_{2}\right)-\exp \left(i\left(\omega_{z 2}-\frac{1}{2} \bar{J}_{12}\right) t_{2}\right)\right]\right\},
\end{aligned}
$$

where $a=-2 \sin \theta \sin \left(\omega_{z 1}-\omega_{z 2}\right) t_{r}$ and $b=\sin 2 \theta\left[1-\cos \left(\omega_{z 1}-\omega_{z 2}\right) t_{r}\right]$. Integration of the absolute square of (5) and of the reference signal with the same $T_{2} \gg \bar{J}_{12}^{-1}$ gives the energy ratio (2) for this case:

$$
R\left(t_{r}\right)=\frac{1}{4}\left\{4 \sin ^{2} \theta \sin ^{2}\left(\omega_{z 1}-\omega_{z 2}\right) t_{r}+\sin ^{2} 2 \theta\left[1-\cos \left(\omega_{1}-\omega_{2}\right) t_{r}\right]^{2}\right\}
$$

which agrees with the numerical calculation in Fig. 2(a). Note that with the assumption of well-resolved lines, $R\left(t_{r}\right)$ is independent of $T_{2}$, but for $1 / T_{2} \gg \bar{J}_{12}$ the phenomenon would be suppressed. The chemical-shift difference is important in that it breaks the symmetry during $t_{r}$, allowing escape from the para manifold, but in the weak-coupling limit, the $J$ coupling sets the time scale for development of signal.

Some further insight can be gained independent of the particular system by consideration of the time-averaged response separately from the oscillatory problem. Specifically, we decompose the density operator as

$$
\sigma\left(t_{r}\right)=\langle\sigma\rangle+\left[\sigma\left(t_{r}\right)-\langle\sigma\rangle\right]
$$

where the quasiconstant $\langle\sigma\rangle$ is the density operator averaged over some period shorter than $T_{1}$ and $T_{2}$, but longer than the period of the oscillations of $\sigma\left(t_{r}\right)$. If the rate of reaction, including the mixing together of reactants, were comparable to or smaller than the frequencies characteristic of $\sigma\left(t_{r}\right)$, then the density operator for the full ensemble of product molecules would in fact tend toward $\langle\sigma\rangle$, the oscillatory terms being eliminated irreversibly by the averaging over the distribution of times since reaction. In such a case, only $\langle\sigma\rangle$ would survive to contribute to any eventual coherent signal.

In general, finding $\langle\sigma\rangle$ requires finding the eigenfunctions of $H$, and so we set this aside and consider a simple estimate of $\langle\sigma\rangle$. In the case of weak coupling typical of liquid-state proton NMR in high fields, then

$$
\langle\sigma\rangle=\frac{1}{4}-I_{z 1} I_{z 2} .
$$

This contains just $3^{-1 / 2}$ of the spin order (norm of the traceless part of $\sigma$ ) which was initially present. The time-averaged density operator (8) is a homonuclear $J$ ordered state. ${ }^{5}$ It differs from those reported previously in being formed not by resonant irradiation, but as the product of a chemical reaction in a magnetic field. Most importantly, it is of order unity, rather than of the order of equilibrium spin polarization. As is well known, such states of bilinear spin ordering are conveniently read out into observable transverse magnetization by a $\pi / 4$ pulse followed by evolution under the spin-spin couplings. ${ }^{5,6}$ The dashed lines in Fig. 2 show the energy ratios $R$ corresponding to just this quasiconstant term. These were
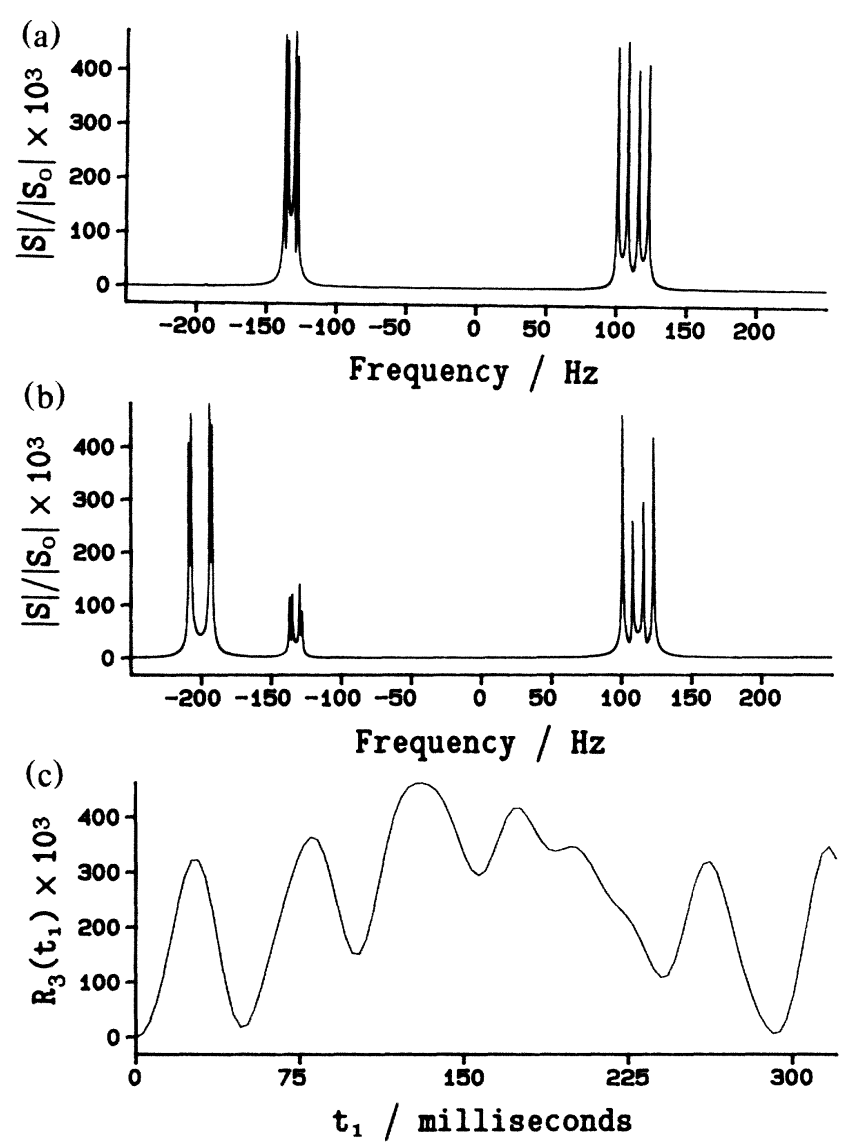

FIG. 3. Bilinear cross polarization on bromoethylene. (a) The magnitude of the spectrum (Fourier transform with respect to $t_{2}$ ) without cross polarization $\left[t_{1}=0\right.$ in Fig. 1(c)]. (b) The corresponding spectrum with $t_{1}=131.2 \mathrm{~ms}$, at which time the multiplet due to proton 3 (cis to the bromine) has grown to a maximum at the expense of resonances due to the protons from $p-\mathrm{H}_{2}$. A Lorentzian broadening of $0.5 \mathrm{~Hz}$ FWHM determines the linewidth. The vertical scale in (a) and (b) is normalized by division of each point of the magnitude spectrum $|S|$, calculated as described, by the maximum peak magnitude $\left|S_{0}\right|$ of the spectrum calculated for the hypothetical case of an ensemble of bromoethylene molecules prepared in their lowest-energy nuclear-spin eigenstates and excited by a $\pi / 2$ pulse. (c) The signal energy of this multiplet as a function of $t_{1}$. The pulse sequence during $t_{1}$ consisted of repetitions of a train of 25 contiguous phase-shifted $\pi$ pulses (Ref. 10), each pulse of length $64 \mu \mathrm{s}$. 
obtained by our numerically projecting out the diagonal terms of $\sigma(0)$ and using only these as the initial condition for the $\pi / 4$ pulse and free-induction decay. The important result is that even under conditions where the chemical reaction events are distributed over many milliseconds (thus averaging away any coherence prior to the first rf pulse), very large signal energies are still anticipated.

Figure 3(a) shows the simulated magnitude spectrum for the $N=3$ product bromoethylene when a $\pi / 4$ pulse acts on $\langle\sigma\rangle$ to elicit the free-induction decay. The amplitude scale is relative to the corresponding spectrum that would be obtained with a $\pi / 2$ pulse and a spin temperature of $0 \mathrm{~K}$. Note that essentially all of the signal intensity is associated with the two protons which had comprised the $p-\mathrm{H}_{2}$ molecule. This will be true whenever these are weakly coupled to other spins.

In most applications it will be desirable to transfer the spin order to other spins to enhance the detectability of a larger number of site-specific resonances. A simple way of doing this is sketched in Fig. $1(\mathrm{c})$. For a period $t_{1}$ a pulse sequence is given which averages away the differences in transition energies between inequivalent spins. One class of such cross-polarization sequences gives an average Hamiltonian containing only scalar operators, ${ }^{7,8}$ which for homonuclei in liquids can be achieved conveniently by a train of contiguous $\pi$ pulses. ${ }^{9}$ The spectrum of Fig. 3(b) was obtained by acting on the timeaveraged initial condition (10) with a particular such train $^{10}$ for a time $t_{1}=131.2 \mathrm{~ms}$ followed by a $\pi / 4 \mathrm{read}$ pulse and detection during $t_{2}$. The intense nonequilibrium magnetization is now shared by the third proton [Fig. 2(b)], which was never part of the $p-\mathrm{H}_{2}$ molecule. The dependence of the signal energy of this new multiplet on the cross-polarization time $t_{1}$ is shown in Fig. 3(c). The plotted quantity $R_{3}\left(t_{1}\right)$ is analogous to the energy ratio introduced in (2), but with numerator and denominator referring only to the multiplet of the third proton. Note that the sharing of spin order occurs prior to the development of any magnetization.

The phenomena predicted here should have wide utility in the study of the chemical reactions of molecules created by hydrogen addition or subsequent reactions. The key requirement is that both hydrogen protons from the $p-\mathrm{H}_{2}$ molecule add together without significant competition from exchange reactions. This is a property of many, but not all, hydrogenations. Facile hydrogen addition requires catalysts, and study of the diverse structures and kinetics in the catalytic or competing reaction paths will be greatly aided by vastly improved NMR sensitivity. This sensitivity could be shared, through cross-polarization techniques, with other nuclei and other molecules, extending the applicability of the method to other problems.

The authors thank the Atlantic Richfield Foundation for partial support of this work.

${ }^{1}$ A. Messiah, Quantum Mechanics (Wiley, New York, 1959), Vol. 2, Chap. 14.

${ }^{2}$ N. Davidson, Statistical Mechanics (McGraw-Hill, New York, 1962), Chap. 9.

${ }^{3}$ U. Fano, Rev. Mod. Phys. 29, 74 (1957).

${ }^{4}$ D. P. Weitekamp, Adv. Magn. Reson. 11, 111 (1983), and references therein-in particular, pp. $158 \mathrm{ff}$.

${ }^{5}$ G. Wagner, G. Bodenhausen, N. Muller, M. Rance, O. W. Sorensen, and R. R. Ernst, J. Am. Chem. Soc. 107, 6440 (1985), and references therein.

${ }^{6} \mathrm{~J}$. Jeener and P. Brockaert, Phys. Rev. 157, 232 (1967).

${ }^{7}$ D. P. Weitekamp, J. R. Garbow, and A. Pines, J. Chem. Phys. 77, 2870 (1982), and 80, 1372 (1984).

${ }^{8}$ L. Braunschweiler and R. R. Ernst, J. Magn. Reson. 53, 521 (1983).

${ }^{9}$ J. S. Waugh, J. Magn. Reson. 68, 189 (1986), and references therein.

${ }^{10}$ R. Tycko and A. Pines, Chem. Phys. Lett. 111, 462 (1985). 